International Journal of Pure and Applied Mathematics

Volume 106 No. $4 \quad 2016,1037-1055$

ISSN: 1311-8080 (printed version); ISSN: 1314-3395 (on-line version)

url: http://www.ijpam.eu

doi: 10.12732/ijpam.v106i4.6

\title{
SOLVING FRACTIONAL INTEGRO DIFFERENTIAL EQUATIONS BY HOMOTOPY ANALYSIS TRANSFORM METHOD
}

\author{
Mohamed S. Mohamed ${ }^{1 \S}$, Muteb R. Alharthi ${ }^{2}$, Refah A. Alotabi ${ }^{3}$ \\ 1,2,3 Mathematics Department \\ Faculty of Science \\ Taif University \\ Hawia, 888 Taif, SAUDI ARABIA \\ ${ }^{1}$ Mathematics Department \\ Faculty of Science \\ Al-Azhar University \\ Nasr City, 11448, Cairo, EGYPT
}

\begin{abstract}
In this paper, we introduce an analytical method, which so called the homotopy analysis transform method (HATM) which is a combination of HAM and Laplace decomposition method (LDM). This scheme is simple to apply linear and nonlinear fractional integro-differential equation and having less computational work in comparison of other exiting methods. The fractional derivatives are described in the Caputo sense. The most useful advantage of this method is to solve the fractional integro-differential equation without using Adomian polynomials and He's polynomials for the computation of nonlinear terms.
\end{abstract}

AMS Subject Classification: 00A71, 45A05, 34A12, 45E10, 7Q10

Key Words: homotopy analysis transforms method, fractional integro-differential equations, Laplace decomposition method

\section{Introduction}

In this paper, we will study the HATM for a special kind of nonlinear fractional

Received: October 17, 2015

Published: March 8, 2016

$\S$ Correspondence author
(C) 2016 Academic Publications, Ltd.

url: www.acadpubl.eu 
integro-differential equation

$$
D^{\alpha} y(t)=p(t) y(t)+g(t)+\lambda \int_{0}^{t} k(t, \tau) F(y(\tau))
$$

and

$$
D^{\alpha} y(t)=p(t) y(t)+g(t)+\lambda \int_{0}^{1} k(t, \tau) F(y(\tau)),
$$

for $t \in[0,1]$ with the initial conditions

$$
y^{(i)}=\delta_{i}, \quad i=0,1,2, \ldots, n-1, n-1<\alpha \leq n, n \in N,
$$

where $g \in L^{2}([0,1]), p \in L^{2}([0,1]), k \in L^{2}\left([0,1]^{2}\right)$ are known functions, $y(t)$ is the unknown function and $D^{\alpha}$ is the Caputo fractional differential operator of order $\alpha$. Such equations arise in the mathematical modeling of various physical phenomena, such as heat conduction in materials with memory. Moreover, these equations are encountered in combined conduction, convection and radiation problems $[1,2]$. In recent years, the analytic results on existence and uniqueness of problems solutions to fractional differential equations have been investigated by many authors [3, 4]. Momani [5] has obtained local and global existence and uniqueness solution of the integro-differential equation. Most of nonlinear fractional integro-differential equations do not have exact analytic solution, so approximation and numerical technique must be used. There are only a few of techniques for the solution of fractional integro- differential equations, since it is relatively a new subject in mathematics. Recently, several numerical methods to solve fractional differential equations and fractional integrodifferential equations have been given. Nawaz [6] employed variational iteration method to solve the problem. Seyed Alizadeh and Domairry [7] presented the homotopy perturbation method for solving integro-differential equations. Also, Momani [8] and Qaralleh [9] applied Adomian polynomials to solve fractional integro-differential equations and systems of fractional integro-differential equations. Zhang and Tang [10] presented homotopy analysis method for higherorder fractional integro-differential equations. Yang [11] applied the hybrid of block- pulse function and Chebyshev polynomials to solve nonlinear Fredholm fractional integro-differential equations. In addition, the applications of collocation method [12-14], wavelet method [15-17] and spectral method [18, 19] for solution of fractional integro-differential equations. The Laplace decomposition method is a numerical algorithm to solve nonlinear ordinary, partial differential equations. Khuri [20] used this method for the approximate solution of a class of nonlinear ordinary differential equations. The numerical technique basically 
illustrates how the Laplace transform can be used to approximate the solution of the nonlinear differential by manipulating the decomposition method which was first introduced by Adomian method.

The main aim of this article is to present analytical and approximate solution of fractional integro-differential equations by using new mathematical tool like homotopy analysis transform method. The proposed method is coupling of the homotopy analysis method HAM and Laplace transform method [2126]. We have studied some of linear and nonlinear fractional integro-differential equations with the help of homotopy analysis transform method.

This paper is organized as follows: We begin by introducing some necessary definitions, mathematical preliminaries of the fractional calculus theory, the Laplace transform and inverse Laplace transform for some functions is demonstrated. In section 3, a short description of the basic ideas of the homotopy analysis method will be stated and homotopy analysis transform method is applied to construct approximate solution. In section 4, the proposed method is applied to several examples. Also, conclusions given in the last section.

\section{Preliminaries and Notations}

In this section, we give some basic definitions and properties of fractional calculus theory which are further used in this paper.

Definition 1. A real function $h(t) t>0$, is said to be in the space $C_{\mu}, \mu \in R$ if there exists a real number $p>\mu$ such that $h(t)=t^{p} h_{1}(t)$ where $h_{1}(t) \epsilon[0, \infty)$ and it is said to be in the space $C_{\mu}^{\mathbf{n}}$ if and only if $h^{(n)} \epsilon C_{\mu}, n \in N$.

Definition 2. The Riemann-Liouville fractional integral operator $\left(J^{\alpha}\right)$ of order $\alpha \geq 0$ of a function $h \in C_{\mu}, \mu \geq-1$ is defined as

$$
\begin{gathered}
J^{\alpha} h(t)=\frac{1}{\Gamma(\alpha)} \int_{0}^{t}(t-\tau)^{\alpha-1} h(t) d \tau \quad(\alpha>-1), \\
J^{0} h(t)=h(t),
\end{gathered}
$$

$\Gamma(\alpha)$ is the well- known Gamma function. Some of the properties of the operator $J^{\alpha}$, which we will need here, are as follows:

(1) $J^{\alpha} J^{\beta} h(t)=J^{\alpha+\beta} h(t)$,

(2) $J^{\alpha} J^{\beta} h(t)=J^{\beta} J^{\alpha} h(t)$,

(3) $J^{\alpha} J^{\gamma}=\frac{\Gamma(\gamma+1)}{\Gamma(\alpha+\gamma+1)} t^{\alpha+\gamma}$ where $\beta \geq 0$ and $\gamma \geq-1$. 
Definition 3. The fractional derivative $\left(D^{\alpha}\right)$ of $h(t)$ in the Caputo's sense is defined as

$$
D^{\alpha} h(t)=\frac{1}{\Gamma(n-\alpha)} \int_{0}^{t}(t-\tau)^{n-\alpha-1} h^{(n)}(\tau) d \tau
$$

for

$$
n-1<\alpha \leq n, \quad n \in N, \quad t>0, \quad h \in C_{-1}^{n} .
$$

The following are two basic properties of Caputo's fractional derivative, see $[4]$.

(1) Let $h \in C_{-1}^{n}, n \in N$. Then $D^{\alpha} h, 0 \leq \alpha \leq n$ is well defined and $D^{\alpha} h \in C_{-1}$.

(2) Let $n-1<\alpha \leq n, n \in N$ and $h \in C_{\mu}^{n}, \mu \geq-1$. Then

$$
\left(J^{\alpha} D^{\alpha}\right) h(t)=h(t)-\sum_{k=0}^{n-1} h^{(k)}\left(0^{+}\right) \frac{t^{k}}{k !} .
$$

Definition 4. The fractional derivative of $f(t)$ in the Caputo sense is defined as

$$
D^{\alpha} f(t)=J^{m-\alpha} D^{m} f(t) .
$$

Here $m-1<\alpha \leq m, \in N, t>0$ and $f \in C_{-1}^{n}$.

In the present work, the fractional derivatives are considered in the Caputo sense. The reason for adopting the Caputo definition, as pointed by [1], is as follows: to solve differential equations (both classical and fractional), we need to specify additional conditions in order to produce a unique solution. For the case of the Caputo fractional differential equations, these additional conditions are just the traditional conditions, which are akin to those of classical differential equations, and are therefore familiar to us. In contrast, for the RiemannLiouville fractional differential equations, these additional conditions constitute certain fractional derivatives (and/or integrals) of the unknown solution at the initial point $x=0$, which are functions of $x$. These initial conditions are not physical; furthermore, it is not clear how such quantities are to be measured from experiment, say, so that they can be appropriately assigned in an analysis $[6]$. 


\subsection{Laplace Operation}

The Laplace transform is a powerful tool in applied mathematics and engineering. The Laplace transform is indispensable in certain areas of control theory. As we will see in later sections we can use Laplace transforms to reduce a differential equation to an algebra problem. The algebra can be messy on occasion, but it will be simpler than actually solving the differential equation directly in many cases. Laplace transforms can also be used to solve IVP's that we can't use any previous method on.

\subsection{Laplace Transform}

Given a function $f(x)$ defined for $0<x<1$, the Laplace transform $F(s)$ is defined as

$$
F(s)=L[f(x)]=\int_{0}^{\infty} f(x) e^{-s x} d x,
$$

at least for those sfor which the integral converges.

Let $f(x)$ be a continuous function on the interval $[0,1)$ which is of exponential order, that is, for some $c \in R$ and $x>0$

$$
\sup \frac{|f(x)|}{e^{c x}}<\infty
$$

In this case the Laplace transform (2.1) exists for all $s>c$.

Lemma 1. Laplace Transform of an Integral If $F(s)=L[f(t)]$ then

$$
L\left[\int_{0}^{t} F(\tau) d \tau\right]=\frac{F(s)}{s} .
$$

Lemma 2. The Laplace transforms of Caputo fraction derivatives for $m-$ $1<\alpha \leq m, m \in N$

$$
L\left[D^{\alpha} f(x)\right]=\frac{s^{m} F(s)-s^{m-1} F(0)-s^{m-2} F^{\prime}(0)-\cdots-f^{(m-1)}(0)}{s^{m-\alpha}} .
$$

Now, we can transform fractional differential equations into algebraic equations and then by solving this algebraic equations, we can obtain the unknown Laplace function $F(s)$. 


\subsection{Inverse Laplace Transform}

The Inverse Laplace Transform of $F(s)$ is defined as:

$$
f(x)=L^{-1}[F(s)]=\frac{1}{2 \pi i} \int_{T-i \sigma}^{T+i \sigma} e^{s x} F(s) d s,
$$

Where $\sigma$ is large enough that $F(s)$ is defined for the real part of $s \geq \sigma$.

\section{Basic Idea of the Fractional Homotopy Analysis Transform Method (FHATM)}

To illustrate the basic idea of the HATM for the fractional partial differential equation, we consider the following fractional partial differential equation as

$$
\begin{gathered}
D_{t}^{n \alpha} y(x, t)+R[x] y(x, t)+N[x] y(x, t)=g(x, t), \\
t>0, \quad x \in R, \quad n-1<n \alpha \leq n,
\end{gathered}
$$

where $D_{t}^{n \alpha}=\frac{\partial^{\text {n } \alpha}}{\partial t^{n \alpha}}, R[x]$ is the linear operator in $x N[x]$ is the general nonlinear operator in $x$, and $g(x, t)$ are continuous functions. For simplicity, we ignore all initial and boundary conditions, which can be treated in similar way. On applying Laplace transform on both sides of (3.1), we get

$$
L\left[D_{t}^{n \alpha} y(x, t)\right]+L[R[x] y(x, t)]+L[N[x] y(x, t)]=L[g(x, t)] .
$$

Now, using the differentiation property of the Laplace transform, we have

$$
\begin{aligned}
& L[y(x, t)] \\
& -\frac{1}{s^{n \alpha}} \sum_{k=0}^{n-1} s^{(n \alpha-k-1)} y^{k}(x, 0) \frac{1}{s^{n \alpha}} L(R[x] y(x, t)+N[x] y(x, t)-g(x, t)) \\
& =0 .
\end{aligned}
$$

We define the nonlinear operator:

$$
\begin{aligned}
N[\varphi(r, t ; q)]=L[\varphi(r, t & ; q)]-\frac{1}{s^{n \alpha}} \sum_{k=0}^{n-1} s^{(n \alpha-k-1)} y^{k}(x, 0) \\
& +\frac{1}{s^{n \alpha}} L(R[x] y(x, t)+N[x] y(x, t)-g(x, t)),
\end{aligned}
$$


where $\mathrm{q} \in[0,1]$ is an embedding parameter and $\varphi(x, t ; q)$ is the real function of $\mathrm{x}, \mathrm{t}$, and $\mathrm{q}$. By means of generalizing the traditional homotopy methods, Liao [18-24] constructed the zero order deformation equation:

$$
\left.(1-q) L[\varphi(x, t ; q)]-y_{0}(x t)\right]=\hbar q H(x t) N[\varphi(x, t ; q)],
$$

where $\hbar$ is a nonzero auxiliary parameter, $H(x, t) \neq 0$ is an auxiliary function, $y_{0}(x t)$ is an initial guess of $y(x, t)$ and $\varphi(x, t ; q)$ is an unknown function. It is important that one has great freedom to choose the auxiliary parameter in HATM. Obviously, when $\mathrm{q}=0$ and $\mathrm{q}=1$, it holds

$$
\varphi(x, t ; 0)=y_{0}(x t), \quad \varphi(x, t ; 1)=y(x, t),
$$

respectively. Thus, as $q$ increases from 0 to 1 , the solution varies from the initial uses $y_{0}(x t)$ to the solution $y(x, t)$. Expanding $\varphi(x, t ; q)$ in Taylor's series with respect to $q$, we have

$$
\varphi(x, t ; q)=y_{0}(x, t)+\sum_{m=1}^{\infty} q^{m} y_{m}(x, t),
$$

where

$$
y_{m}(x, t)=\frac{1}{m !} \frac{\partial^{m} ?(x, t ; q)}{\partial q^{m}} \mid q=0 .
$$

The convergence of series solution (3.7) is controlled by $\hbar$. If the auxiliary linear operator $\mathrm{R}$, the initial guess $y_{0}(x, t)$, the auxiliary parameter $\hbar$, and the auxiliary function $\mathrm{H}$ are properly chosen, and the series (3.7) converges at $\mathrm{q}=$ 1 , then we have

$$
y(x, t)=y_{0}(x, t)+\sum_{m=1}^{\infty} y_{m}(x, t),
$$

which must be one of the solutions of original nonlinear equations. The above expression provides us with a relationship between the initial guess $y_{0}(x, t)$ and the exact solution $y(x t)$ by means of the terms $y_{m}(x, t)(m=1,2,3, \ldots)$, which are still to be determined. We define the vector as

$$
\vec{y}_{n}=\left\{y_{0}(x, t), y_{1}(x, t), y_{2}(x, t), \quad y_{n}(x, t)\right\} .
$$

Differentiating the zero-order deformation equation (3.5) $\mathrm{m}$ times with respect to embedding parameter $\mathrm{q}$ and then setting $q=0$ and finally dividing them by $m$ !, we obtain the $\mathrm{m}$-th order deformation equation:

$$
L\left[y_{m}(x, t)-\chi_{m} y_{m-1}(x, t)\right]=\hbar q H(x, t) R_{m}\left(\vec{y}_{m-1}, x, t\right) .
$$


Applying the inverse Laplace transform on both sides, we get

$$
y_{m}(x, t)=\chi_{m} y_{m-1}(x, t)+\hbar q L^{-1}\left[H(x, t) R_{m}\left(\vec{y}_{m-1}, x, t\right)\right] .
$$

Here:

$$
R_{m}\left(\vec{y}_{m-1}, x, t\right)=\frac{1}{m !} \frac{\partial^{m}(x, t ; q)}{\partial q^{m}} \mid q=0
$$

and

$$
\chi_{m}= \begin{cases}0, & m \leq 1, \\ 1, & m>1 .\end{cases}
$$

Applying the inverse Laplace transform on both sides of equation (3.11), we have

$$
\begin{aligned}
y_{m}(x, t) & =\chi_{m} y_{m-1}(x, t) \\
& -\chi_{m} \sum_{k=0}^{n-1} s^{(n \alpha-k-1)} y^{k}(x, 0)+\hbar H(x, t) L^{-1}\left[R_{m}\left(\vec{y}_{m-1}, x, t\right)\right] .
\end{aligned}
$$

It is easy to obtain $y_{m}(x, t)$ for $\mathrm{m} \geq 1$, and at $m$-th order, we have

$$
y_{m}(x, t)=\sum_{m=0}^{M} y_{m}(x, t),
$$

where $M \rightarrow \infty$, we get an accurate approximation of the original equation (3.1). The solution of problem (3.1) is obtained by putting these $y_{m}(x, t)$ 's in (3.9) and choosing a suitable value of $\hbar$ for the convergence of the series.

\section{Illustrative Examples}

In this section, we discuss the implementation of our proposed method and investigate its accuracy by applying the HAM with coupling of the Laplace transform method. The simplicity and accuracy of the proposed algorithm is illustrated through the following Illustrative examples. All the results are calculated by using the symbolic calculus software Mathematic.

Example 4.1. First, we consider the following fractional integro-differential equation, for $t \in I=[0,1]$.

$$
D^{\alpha} u(t)=u(t)+\frac{8}{3 \Gamma(0.5)} t^{1.5}-t^{2}-\frac{1}{3} t^{3}+\int_{0}^{t} u(\mu) d \mu,
$$




$$
u(0)=0,
$$

The exact solution is $u(t)=t^{2}$, that is for $\alpha=0.5$

Applying the Laplace transform on both sides in (4.1.1) and after using the differentiation property of Laplace transform, we get:

$$
\begin{gathered}
s^{\alpha} L[u(t)]-s^{\alpha-1} u(0)-L[u(t)]+L\left(\frac{-8}{3 \Gamma(0.5)} t^{1.5}+t^{2}+\frac{1}{3} t^{3}\right)-\frac{1}{s} L[u(t)]=0, \\
L[u(t)]-\frac{1}{s^{\alpha}} L[u(t)]+\frac{1}{s^{\alpha}}\left(\frac{-8}{3 \Gamma(0.5)}\left(\frac{(1.5) !}{s^{2.5}}\right)+\frac{2}{s^{3}}+\frac{1}{3}\left(\frac{3 !}{s^{4}}\right)\right) \\
-\frac{1}{s} \frac{1}{s^{\alpha}} L[u(t)]=0 .
\end{gathered}
$$

We now define a nonlinear operator as:

$$
\begin{aligned}
& N[\varphi(t, s ; q)]=L[\varphi(t, s ; q)]-\frac{1}{s^{\alpha}} L[\varphi(t, s ; q)] \\
& +\frac{1}{s^{\alpha}}\left(\frac{-8}{3 \Gamma(0.5)}\left(\frac{(1.5) !}{s^{2.5}}\right)+\frac{2}{s^{3}}+\frac{1}{3}\left(\frac{3 !}{s^{4}}\right)\right)-\frac{1}{s} \frac{1}{s^{\alpha}} L[\varphi(t, s ; q)]=0 .
\end{aligned}
$$

Using the above definition, with assumption $H(t)=1$, we construct the zeroth-order deformation equation:

$$
\left.(1-q) L[\varphi(t, s ; q)]-u_{0}(t)\right]=\hbar q H(t) N[\varphi(t, s ; q)] .
$$

Obviously, when $q=0$ and $q=1$ :

$$
\varphi(t ; 0)=u_{0}(t), \quad \varphi(t ; 1)=u(t) .
$$

Thus, we obtain the $m$-th order deformation equation:

$$
L\left[u_{m}(t)-\chi_{m} u_{m-1}(t)\right]=\hbar R_{m}\left(\vec{u}_{m-1}, t\right) .
$$

Operating the inverse Laplace transform on both sides in (4.1.6), we get

$$
u_{m}(t)=\chi_{m} u_{m-1}(t)+\hbar L^{-1}\left[R_{m}\left(\vec{u}_{m-1}, t\right)\right] .
$$

Here

$$
R_{m}\left(\vec{u}_{m-1}\right)=L\left[u_{m-1}(t)\right]-\frac{1}{s^{\alpha}} L\left[u_{m-1}(t)\right]
$$




$$
+\frac{1}{s^{\alpha}}\left(\frac{-8}{3 \Gamma(0.5)}\left(\frac{(1.5) !}{s^{2.5}}\right)+\frac{2}{s^{3}}+\frac{1}{3}\left(\frac{3 !}{s^{4}}\right)\right)-\frac{1}{s} \frac{1}{s^{\alpha}} L\left[u_{m-1}(t)\right]=0 .
$$

The solution of $m$-th order deformation equation (4.1.7) is given as

$$
\begin{aligned}
& u_{m}(t)=\chi_{m} u_{m-1}(t) \hbar L^{-1}\left[L\left[u_{m-1}(t)\right]-\frac{1}{s^{\alpha}} L\left[u_{m-1}(t)\right]\right. \\
& \left.\quad+\frac{1}{s^{\alpha}}\left(\frac{-8}{3 \Gamma(0.5)}\left(\frac{(1.5) !}{s^{2.5}}\right)+\frac{2}{s^{3}}+\frac{1}{3}\left(\frac{3 !}{s^{4}}\right)\right)-\frac{1}{s} \frac{1}{s^{\alpha}} L\left[u_{m-1}(t)\right]\right]
\end{aligned}
$$

Using the initial approximation $u_{0}(t)=u(0)=0$ and the iterative scheme (4.1.9), we obtain the various iterates:

$$
\begin{gathered}
u_{1}(t)=-\frac{2 h t^{1.5+\alpha}}{\Gamma(2.5+\alpha)}+\frac{2 h t^{2+\alpha}}{\Gamma(3+\alpha)}+\frac{2 h t^{3+\alpha}}{\Gamma(4+\alpha)} \\
u_{2}(t)=2 h t^{2+\alpha}\left(\frac{1+h}{\Gamma(3+\alpha)}-\frac{h t^{\alpha}}{\Gamma(3+2 \alpha)}\right)+2 h t^{3+\alpha}\left(\frac{1+h}{\Gamma(4+\alpha)}-\frac{2 h t^{\alpha}}{\Gamma(4+2 \alpha)}\right) \\
+h\left(-\frac{2 h^{2} t^{4+2 \alpha}}{\Gamma(5+2 \alpha)}\right) \\
\left.\Gamma\left(-\frac{2 t^{1.5+\alpha}}{\Gamma(2.5+\alpha)}+t^{2 \alpha}\left(\frac{2 t^{1.5}}{\Gamma(2.5+2 \alpha)}+\frac{2 t^{2.5}}{\Gamma(3.5+2 \alpha)}\right)\right)\right) .
\end{gathered}
$$

Setting $\alpha=0.5$ in (4.1.10) and $\hbar=-1$, we get:

$u_{1}(t)=t^{2}-0.601802 t^{2.5}-0.171943 t^{3.5}$,

$u_{2}(t)=t^{2}+0.601802 t^{2.5}-0.333333 t^{3}+0.171943 t^{3.5}-0.166667 t^{4}-0.0166667 t^{5}$,

Then, the HATM series solution is

$$
\begin{gathered}
\varphi_{n}(t)=\sum_{\mathrm{i}=0}^{n-1} u_{\mathrm{i}}(t)=u_{1}+u_{2}+\cdots, \quad n=1,2, \ldots \\
\begin{array}{c}
u(t)=t^{2}-0.08333 t^{4}-0.06667 t^{5}-0.016666 t^{6} \\
-0.0015873 t^{7}-0.0000496 t^{8},
\end{array}
\end{gathered}
$$




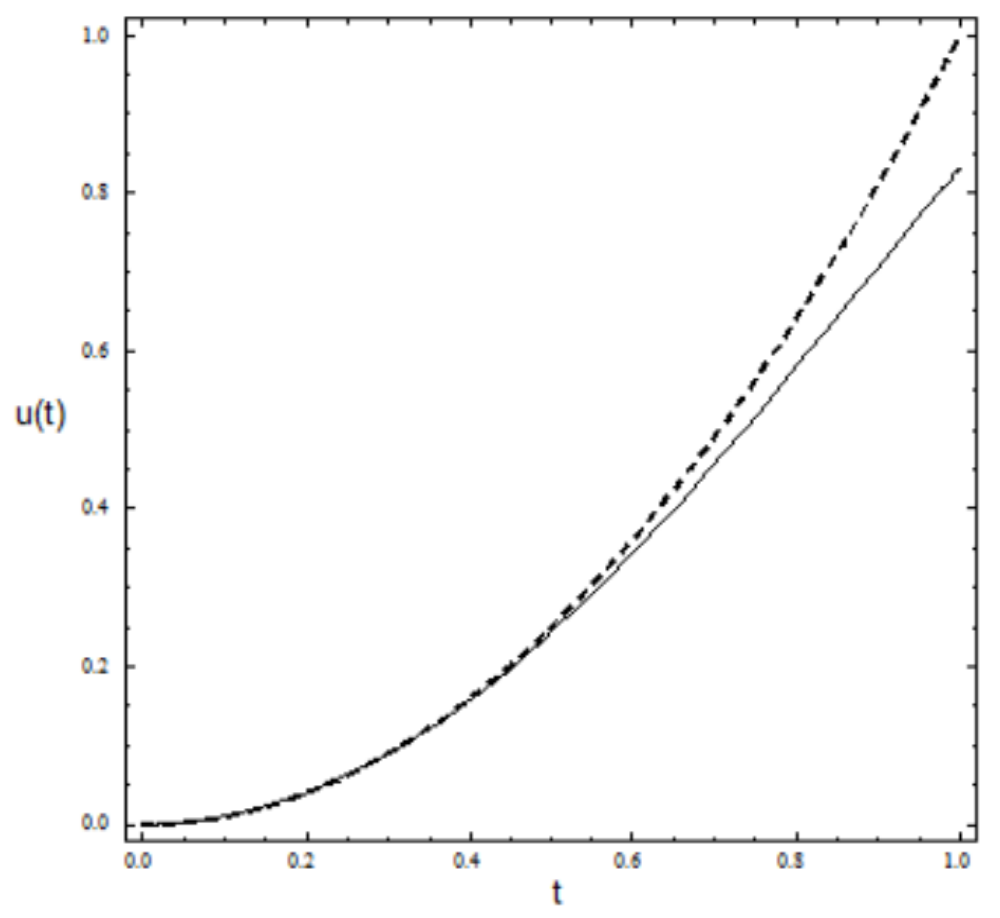

Figure 1: (Black online) The exact solution (4.1.12) is compared with the approximate solution HATM (4.1.11)

$$
u(t)=\varphi_{n}(t)=\sum_{\mathrm{i}=0}^{n-1} u_{\mathrm{i}}(t)=t^{2}
$$

Example 4.2. Consider the following fractional integro-differential equation, for $x \in[0,1]$

$$
\begin{gathered}
D^{\alpha} u(t)=-1+\int_{0}^{t} u^{2}(\mu) d \mu, \quad 0<\alpha \leq 1, \\
u(0)=0,
\end{gathered}
$$

which has the exact solution $u(t)=\left(\frac{1}{28} t^{4}-x\right) /\left(\frac{1}{28} t^{3}+1\right)$, that is, for $\alpha=1$.

Applying the Laplace transform on both sides in Eq. (4.2.13) and after using the differentiation property of Laplace transform, we get:

$$
s^{\alpha} L[u(t)]+s^{\alpha-1} u(0)+L[1]-\frac{1}{s} L\left[u^{2}(t)\right]=0
$$




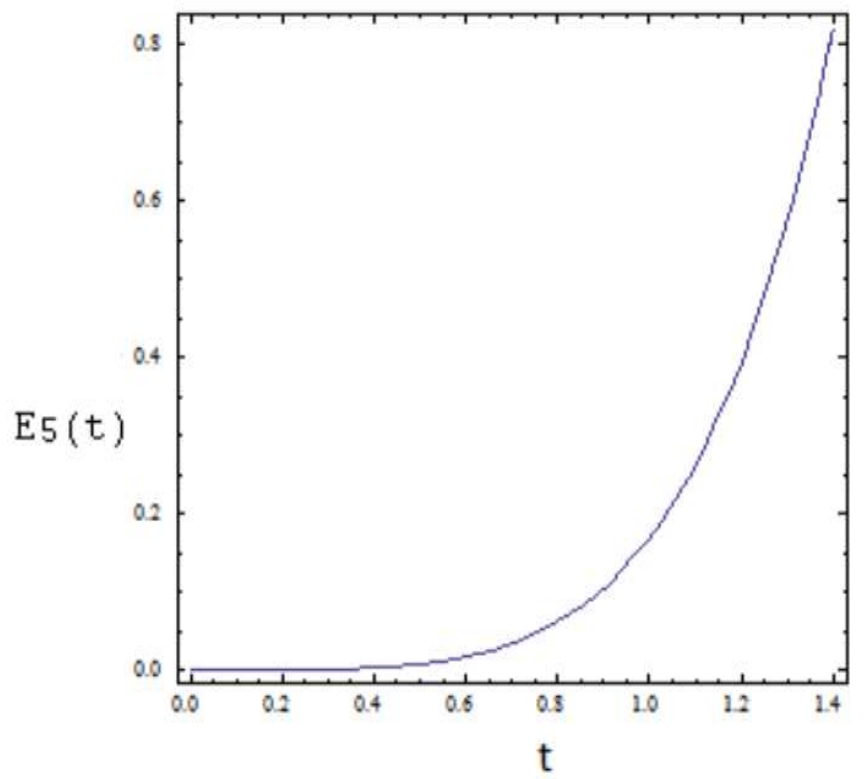

Figure 2: Figure (2) : The absolute error of $u_{5}$ and exact solution of (4.1.12).

$$
L[u(t)]+\frac{1}{s^{\alpha}}\left(\frac{1}{s}\right)-\frac{1}{s} \frac{1}{s^{\alpha}} L\left[u^{2}(t)\right]=0 .
$$

We now define a nonlinear operator as:

$$
N[\varphi(x, t ; q)]=L[\varphi(x, t ; q)]+\frac{1}{s^{\alpha}}\left(\frac{1}{s}\right)-\frac{1}{s} \frac{1}{s^{\alpha}} L\left[\varphi^{2}(x, t ; q)\right]=0
$$

Using the above definition, with assumption $H(x)=1$, we construct the zerothorder deformation equation:

$$
\left.(1-q) L[\varphi(x, t ; q)]-u_{0}(x)\right]=\hbar q H(x) N[\varphi(x, t ; q)]
$$

Obviously, when $\mathrm{q}=0$ and $\mathrm{q}=1$,

$$
\varphi(t ; 0)=u_{0}(t), \quad \varphi(x ; 1)=u(t)
$$

Thus, we obtain the mth-order deformation equation:

$$
L\left[u_{m}(t)-\chi_{m} u_{m-1}(t)\right]=\hbar R_{m}\left(\vec{u}_{m-1}, t\right)
$$




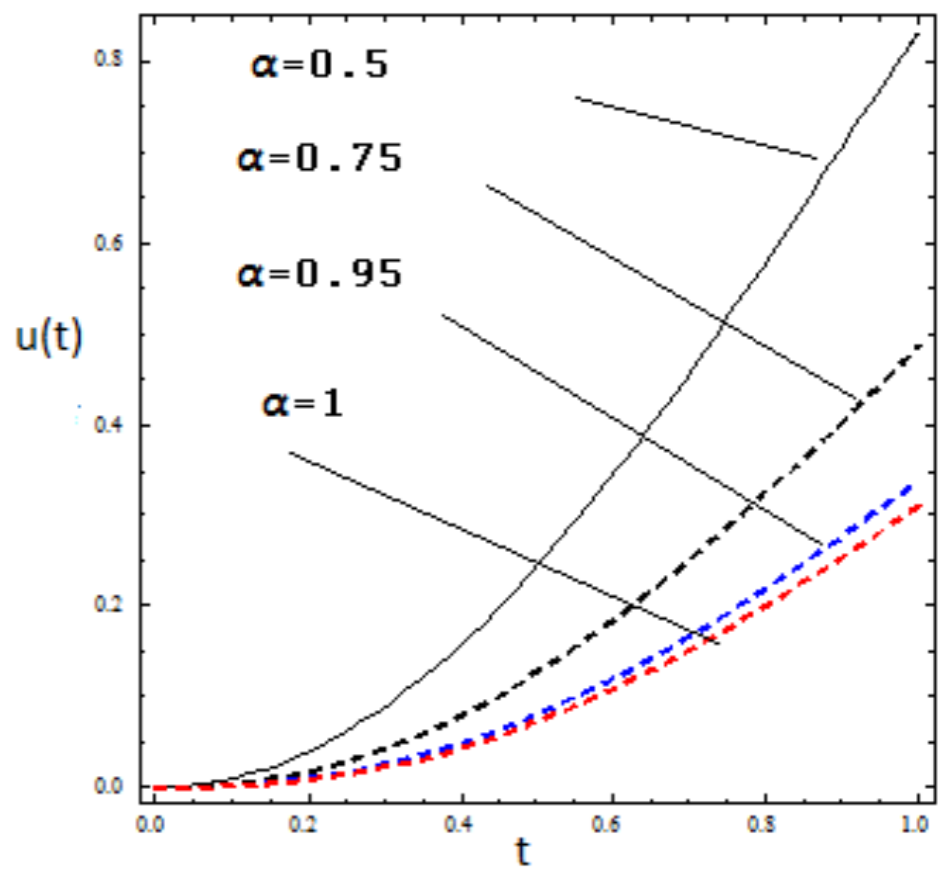

Figure 3: Five-order HATM solution of eq. (4.1.10) for different values of $\alpha$ at $\hbar=-1$

Operating the inverse Laplace transform on both sides in (4.2.18), we get:

$$
u_{m}(t)=\chi_{m} u_{m-1}(t)+\hbar L^{-1}\left[R_{m}\left(\vec{u}_{m-1}, t\right)\right]
$$

Where

$$
R_{m}\left(\vec{u}_{m-1}\right)=L\left[u_{m-1}(t)\right]+\frac{1}{s^{\alpha}}\left(\frac{1}{s}\right)-\frac{1}{s} \frac{1}{s^{\alpha}} L\left[u_{m-1}^{2}(t)\right]=0
$$

Now the solution of mth-order deformation equation (4.2.19) is given as:

$$
u_{m}(t)=\chi_{m} u_{m-1}(t)+\hbar L^{-1}\left[L\left[u_{m-1}(t)\right]+\frac{1}{s^{\alpha}}\left(\frac{1}{s}\right)-\frac{1}{s} \frac{1}{s^{\alpha}} L\left[u_{m-1}^{2}(t)\right]\right]
$$

Using the initial approximation $u_{0}(t)=u(0)=0$ and the iterative scheme (4.2.21), we obtain the various iterates:

$$
u_{1}(t)=\frac{h t^{\alpha}}{\Gamma(1+\alpha)}
$$




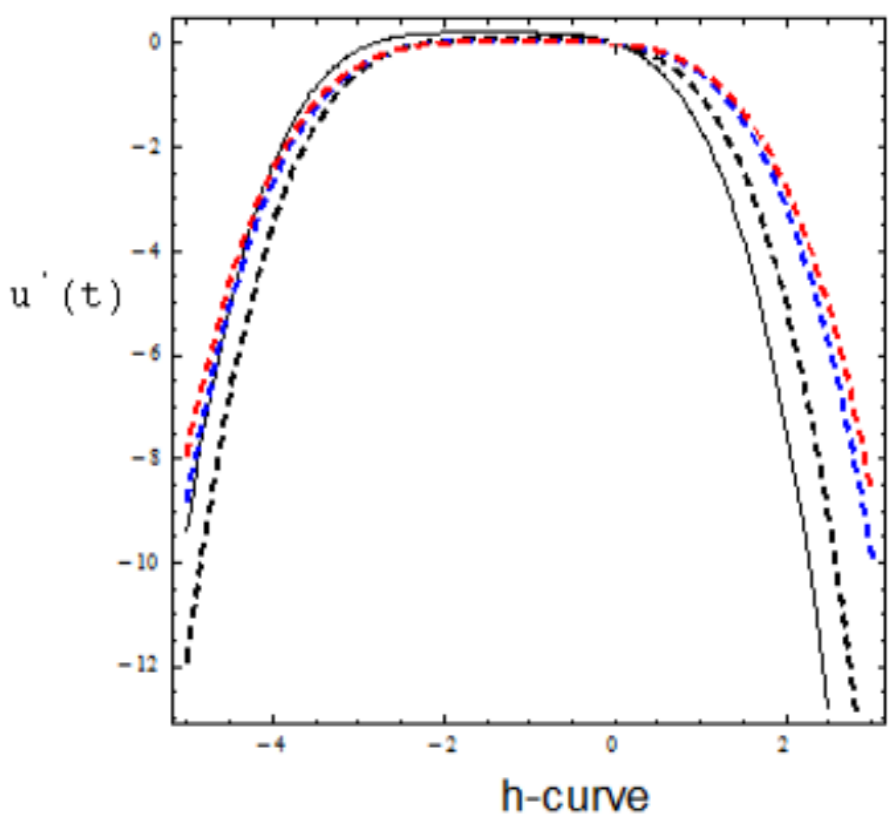

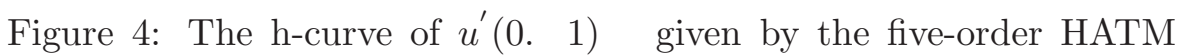
approximate. Solution for different values of $\alpha$

$$
\begin{gathered}
u_{2}(t)=\frac{h(1+h) t^{\alpha}}{\Gamma(1+\alpha)} \\
u_{3}(x)=\frac{h(1+h)^{2} t^{\alpha}}{\Gamma(1+\alpha)}-\frac{h^{3} t^{1+3 \alpha} \Gamma(1+2 \alpha)}{\Gamma(2+3 \alpha) \Gamma(1+\alpha)^{2}}:
\end{gathered}
$$

Setting $\alpha=1$ in (4.2.22) and $\hbar=-1$,we get:

$$
\begin{gathered}
u_{1}(x)=-x \\
u_{2}(x)=0, \\
u_{3}(x)=\frac{x^{4}}{12}
\end{gathered}
$$

Then, the HATM series solution is:

$$
\varphi_{n}(x)=\sum_{\mathrm{i}=0}^{n-1} u_{\mathrm{i}}(x)=u_{1}+u_{2}+\ldots, \quad \mathrm{n}=12 \ldots
$$




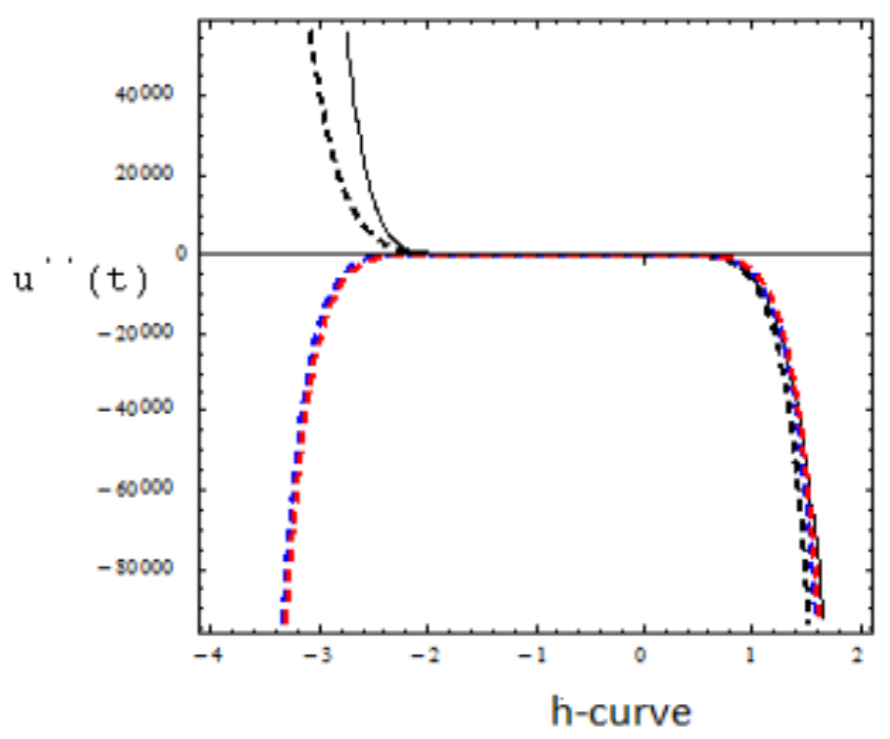

Figure 5: The h-curve of $u^{\prime \prime}(0.75)$ given by the tenth-order HATM approximate. Solution for different values of $\alpha$.

$$
u(x)=\varphi_{n}(x)=\sum_{\mathrm{i}=0}^{n-1} u_{\mathrm{i}}(x)=\left(\frac{1}{28} x^{4}-x\right) /\left(\frac{1}{28} x^{3}+1\right)
$$

\section{Conclusion}

This paper develops an effective and new modification of HAM, which is coupled with homotopy analysis and Laplace transform method, and studied its validity in a wide range with three examples of fractional equations. The method gives more realistic series solutions that converge very rapidly in fractional equations. It is worth mentioning that the method is capable of reducing the volume of the computational work as compared to the classical methods with high accuracy of the numerical result and will considerably benefit mathematicians and scientists working in the field of fractional calculus. It may be concluded that the HATM is very powerful and efficient in finding the approximate solutions as well as analytical solutions of many physical problems arising in sciences and engineering. 


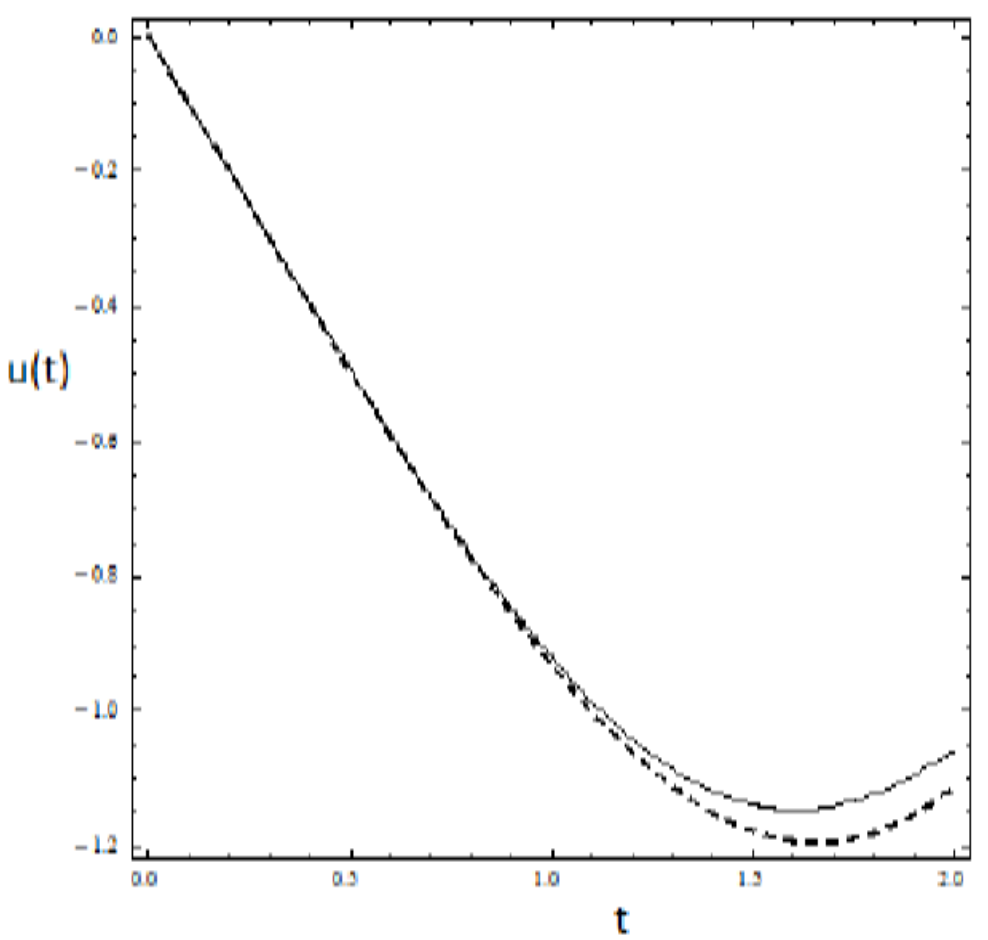

Figure 6: (Black online) The exact solution (4.1.12) is compared with the approximate solution HATM (4.1.11)

\section{References}

[1] M. Caputo, Linear models of dissipation whose Q is almost frequency in dependent-II, Geophys.J. Roy. Astron. Soc., 13(1967), 529-539.

[2] W. E. Olmstead and R. A. Handelsman, Diffusion in a semi-infinite region with nonlinear surface dissipation, SIAM Rev., 18(1976), 275-291.

[3] K. Diethem and N. J. Ford, Analysis of fractional differential equation, J. Math. Anal. Appl., 256(2002), 229-248.

[4] I. Podlubny, Fractional Differential Equations, Academic Press, New York, 1999.

[5] S. M. Momani, Local and global existence theorems on fractional integro-differential equations, J. Fract. Calc., 18(2000), 81-86.

[6] Y. Nawaz, Variational iteration method and homotopy perturbation method for fourthorder fractional integro-differential equations, Comput. Math. Appl., 61(2011), 23302341.

[7] S. R. Seyed Alizadeh, G. G. Domairry and S. Karimpour, An approximation of the analytical solution of the linear and nonlinear integro-differential equations by homotopy perturbationmethod, Acta. Appl. Math., 104(2008), 355- 366. 


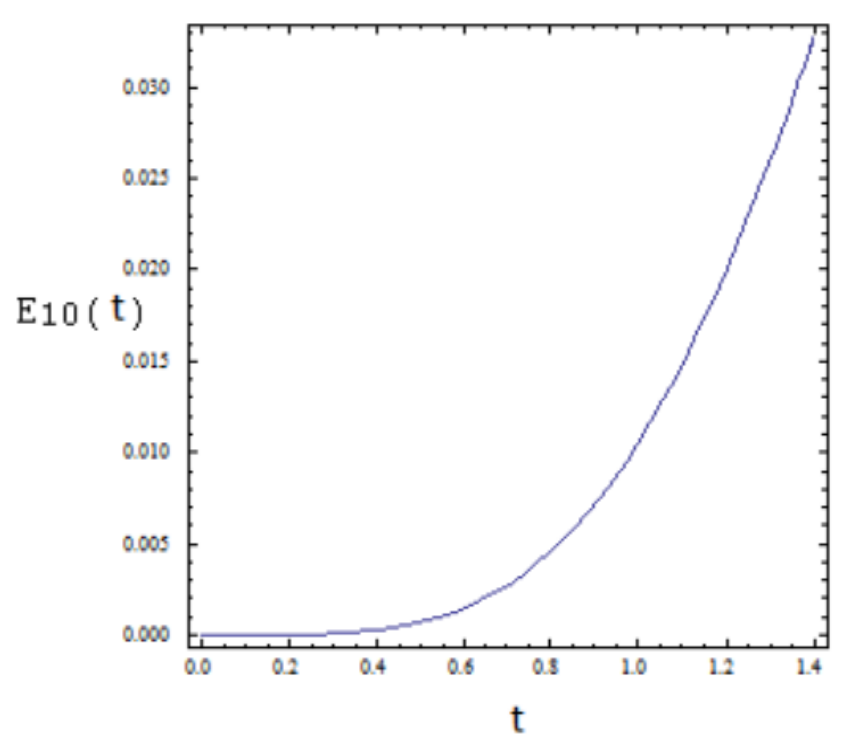

Figure 7: The absolute error of $u_{10}$ and exact solution of (4.2.25).

[8] S. Momani and M. A. Noor, Numerical methods for four-order fractional integrodifferential equations, Appl. Math. Comput., 182(2006), 754-760.

[9] S. Momani and R. Qaralleh, An efficient method for solving systems of fractional integrodifferential equations, Comput. Math. Appl., 52(2006), 459-470.

[10] X. Zhang, B. Tang and Y. He, Homotopy analysis method for higher-order fractional integro-differential equations, Comput. math. appl., 62(2011), 3194-3203.

[11] C. Yang, Numerical solution of nonlinear Fredholm integrodifferential equations of fractional order by using hybrid of block-pulse functions and Chebyshev polynomials, Math. Probl. Eng., ( 2011), 1-11.

[12] E. A. Rawashdeh, Numerical solution of fractional integro-differential equations by collocation method, Appl. math. Comput., 176 (2006), 1-6.

[13] A. Saadatmandi and M. Dehghan, A Legendre collocation method for fractional integrodifferential equations, J. Vib. Control, 17(2011), 2050-2058.

[14] X. Ma and C. Huang, Numerical solution of fractional integro-differential equations by a hybrid collocation methodq, Appl. Math. Comput., 219 (2013), 6750-6760.

[15] H. Saeedi and M. Mohseni Moghadam, Numerical solution of nonlinear Volterra integrodifferential equations of arbitrary order by CAS wavelets, Commun. Nonlinear. Sci. Numer. Simulat., 16(2011), 1216-1226.

[16] L. Zhu and Q. Fan, Solving fractional nonlinear Fredholm integro-differential equations by the second kind Chebyshev wavelet, Commun. Nonlinear. Sci. Numer. Simulat., 17(2012), 2333-2341. 


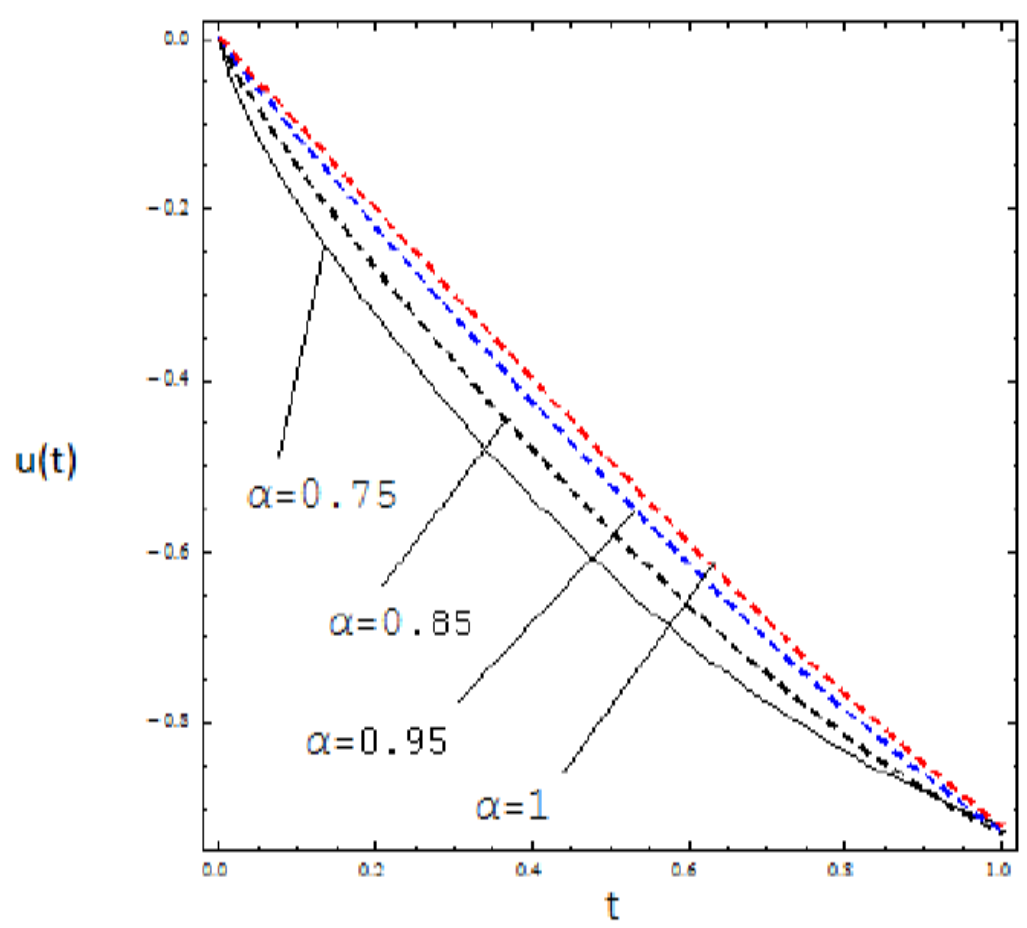

Figure 8: Tenth-order HATM solution of eq. (4.2.24) for different values of $\alpha$ at $\hbar=-1$.

[17] L. Zhu and Q. Fan, Numerical solution of nonlinear fractional-order Volterra integrodifferential equations by SCW, Commun. Non linear. Sci. Numer. Simulat., 18(2013), 1203-1213.

[18] P. Mokhtary and F. Ghoreishi, The L2 convergence of the Legendre spectral Tau matrix formulation for nonlinear fractional integro differential equations, Numer. Algor., 58( 2011), 475-496.

[19] M. M. Khader and N. H. Sweilam, On the approximate solutions for system of fractional integro-differential equations using Chebyshev pseudo-spectral method, Appl. Math. Model, 37(2013), 9819-9828.

[20] S. A. Khuri, A Laplace decomposition algorithm applied to a class of nonlinear differential equations, J. Math. Appl., 1( 2001), 141-155.

[21] Y. Khan, N. Faraz, S. Kumar, A. A.Yildirim. A coupling method of homotopy method and Laplace transform for fractional modells. UPB Sci Bull Ser A Appl Math Phys., $74(1)(2012), 57-68$.

[22] J. Singh, D. Kumar, S. Kumar. New homotopy analysis transform algorithm to solve Volterra integral equation. Ain Shams Eng J 2013, doi: .org/10.1016/j.asej.2013.07.004. 
[23] M. S. Mohamed, Khaled A. Gepreel, Faisal Al-Malki, Maha Al-humyani, Approximate solutions of the generalized Abel's integral equations using the extension Khan's homotopy analysis transformation method, Journal of Applied Mathematics. ( Hindawi Publishing Corporation) (Volume 2015, Article ID 357861, 9 pages).

[24] M. S. Mohamed, Analytical treatment of Abel integral equations by optimal homotopy analysis transform method, Journal of Information and Computing Science; 10(1) (2015): 19-28.

[25] M. S. Mohamed, Faisal Al-Malki, Maha Al-humyani, A General Algorithm for Solving the Fractional Order Partial Differential Equation for Predator-Prey by Homotopy Analysis Transform Method, jokull journal. 64(10)(2014): 167-176.

[26] M. S. Mohamed, Faisal Al-Malki, Maha Al-humyani, Homotopy Analysis Transform Method for Time-Space fractional gas dynamics equation, Journal of Gen. Math. Notes. (24)(1)(2014): 1:16. 
